\title{
Clinical and genetic characteristics of Japanese nephronophthisis patients
}

\author{
Keisuke Sugimoto $^{1}$ - Tomoki Miyazawa ${ }^{1} \cdot$ Takuji Enya $^{1} \cdot$ Hitomi Nishi $^{1} \cdot$ \\ Kohei Miyazaki $^{1} \cdot$ Mitsuru Okada $^{1} \cdot$ Tsukasa Takemura $^{1}$
}

Received: 30 August 2015/Accepted: 4 October 2015/Published online: 23 October 2015

(C) Japanese Society of Nephrology 2015

\begin{abstract}
Background Nephronophthisis (NPH) accounts for 4-5\% of end-stage renal disease occurring in childhood.

Method We investigated the clinical context and characteristics of renal and extrarenal symptoms, as well as the $N P H P$ genes, in 35 Japanese patients with clinical and histologic features suggesting NPH.

Results NPH occurred fairly uniformly throughout Japan irrespective of region or gender. In three families, NPH affected siblings. The median age of patients was 12.5 years. Renal abnormalities attributable to NPH discovered through mass screening, such as urine tests in school. However, NPH accounted for less than $50 \%$ of children with abnormal findings, including incidentally discovered renal dysfunction during evaluation of extrarenal symptoms or during routine check-ups. Typical extrarenal manifestations leaded to discovery including anemia and delayed physical development. The urine often showed low gravity specific density and low molecular weight proteinuria. Frequent renal histologic findings included cystic dilation of tubules, mainly in the medulla, and irregularity of tubular basement membranes. Genetically abnormalities of NPHP1 were not common, with large deletions frequently noted. Compound heterozygotes showing single abnormalities in each of NPHP1, NPHP3, and NPHP4 were observed.

Conclusions Our findings resemble those reported in Western populations.
\end{abstract}

Keisuke Sugimoto

ksugimo@med.kindai.ac.jp

1 Department of Pediatrics, Kinki University Faculty of Medicine, 377-2 Ohno-higashi, Osaka-Sayama 589-8511, Japan
Keywords End-stage renal disease $\cdot$ Renal cysts $\cdot N P H P$ genes $\cdot$ Children $\cdot$ Renal tubules

\section{Introduction}

Nephronophthisis (NPH) is a disease characterized by renal medullary cyst formation. Additional histologic findings include tubulointerstitial nephritis accompanied by progressive sclerosis and hyaline glomeruli. Although NPH characteristically shows autosomal recessive inheritance, it may occur sporadically [1]. NPH accounts for approximately $4-5 \%$ of end-stage renal disease (ESRD) in childhood. Disease subtypes include: infantile NPH (NPH2), which progresses to ESRD around the age of 5 years; juvenile NPH (NPH1), which develops from early childhood to school age and usually progresses to ESRD by an age of about 13 or 14 years; and adolescent NPH (NPH3), with development of ESRD at an average age of 19 years. Juvenile NPH is reported to be the most common subtype [1].

NPHP 1, the gene most often responsible for juvenile nephronophthisis, encodes the nephrocystin-1 molecule. This gene has an extent of approximately $11 \mathrm{kbp}$, and is located on chromosome 2q12-13 [2]. The nephrocystin-1 protein consists of 677 amino acids and includes three coiled domains; two highly acidic negatively charged glutamic acid-rich domains; and an Src-homology 3 domain. Nephrocystin-1 has a molecular weight of $83 \mathrm{kD}$. As this protein is located in the transition zone of primary cilia of renal tubular epithelial cells, its abnormalities typically cause dysfunction of these primary cilia (ciliopathy) $[1,2]$.

NPHP4, whose abnormalities cause a second form of $\mathrm{NPH} 1$, is located on chromosome $1 \mathrm{p} 36$ and encodes the nephrocystin-4 (nephroretinin) molecule. Nephrocystin-4 
Primers for NPHP1

\begin{tabular}{|c|c|c|c|c|c|}
\hline exon & F primer & $5^{\prime}$-nucleotide sequence $-3^{\prime}$ & Rprimer & $5^{\prime}$-nucleotide sequence $-3^{\prime}$ & $\begin{array}{c}\text { Amprified } \\
\text { fragment } \\
\text { length (bp) }\end{array}$ \\
\hline exon1 & NPHP1E01F010 & GACCACCGCAAGAGAACATTT & NPHP1E01R010 & AAGCTCCCAGGATTAGGTGGG & 319 \\
\hline exon2 & NPHP1E02F010 & GGTATATGGGTTITCACTGTA & NPHP1E02R010 & TTCCATTTGATTCCAAAGGAC & 319 \\
\hline exon3 & NPHP1E03F010 & TAATTGCCTTGCCTGCTCAAC & NPHP1E03R010 & CAGACTTAGCAAGCCTGTTCG & 320 \\
\hline exon4 & NPHP1E04F010 & GATAGGTGTAATGTCACACTG & NPHP1E04R010 & CATGGGATCTAACACCTTCTA & 418 \\
\hline exon5 & NPHP1E05F010 & CCAGCTCCAAATATGGGATAT & NPHP1E05R010 & CAGGTGTACAGGCAGAGTTTTC & 380 \\
\hline exon6 & NPHP1E06F010 & GGGAAGCTTITGATAAACCTT & NPHP1E06R010 & GTCATTCACTAGTCAACTGAC & 349 \\
\hline exon7 & NPHP1E07F010 & GTITTGTITTTACTGGAGGG & NPHP1E07R010 & GTTGTCTCCATTTCAAGAAAG & 306 \\
\hline exon8 & NPHP1E08F010 & CTCGTTTTCATCTGGAAACTG & NPHP1E08R010 & GGAAAGCAGGATCAATGAGAA & 443 \\
\hline exon9 & NPHP1E09F010 & СTTCCACTAAAGTCTGTATGT & NPHP1E09R010 & GTGAGATTCAACATCTTCTTC & 322 \\
\hline exon10 & NPHP1E10F010 & TITGGAAGTGCCTGTACTCTA & NPHP1E10R010 & GTCCAAATTCTGCCTTAGTTA & 360 \\
\hline exon11 & NPHP1E11F010 & GCCTGCCAATATTTATTGTTCC & NPHP1E11R010 & TACTCTCTTGGGAATTGGGGA & 494 \\
\hline exon12 & NPHP1E12F010 & TCCTCACTTAGTGTAGCCACT & NPHP1E12R010 & GTCCTCAAAGAACACCAAAGA & 302 \\
\hline exon13 & NPHP1E13F010 & CACCTTCAACATTGGGATTAC & NPHP1E13R010 & CATTCTCATTCCTCAAGGGAT & 365 \\
\hline exon14 & NPHP1E14F010 & GCAAAATGAGATTCTACTGTG & NPHP1E14R010 & AGTTATTGGCATGCTCATAGA & 342 \\
\hline exon15 & NPHP1E15F010 & GGCATAAATGAAATGTCTGAG & NPHP1E15R010 & GTCTCATATGTGTTACCAAGA & 374 \\
\hline exon16 & NPHP1E16F010 & GCACTACTGGGTGGTATATTT & NPHP1E16R010 & GGGAAGAATTAAAGAGGACAA & 330 \\
\hline exon17 & NPHP1E17F010 & GAAGCAAAATTTGGGACTGTT & NPHP1E17R010 & AAAGTCACAACCAGAAACAGA & 316 \\
\hline exon18 & NPHP1E18F010 & CCTAGAAGTCAAAGTGTGTAG & NPHP1E18R010 & GGAGACATCATCCTAGTAACA & 326 \\
\hline exon19 & NPHP1E19F010 & CAGCATTITTAACCCTGTCCA & NPHP1E19R010 & GGGATTATGACTATGGCTACT & 261 \\
\hline exon20 & NPHP1E20F010 & СССТCСАTCСТACСТCTTAGG & NPHP1E20R010 & CTAAGTTGTAAAGTGACAGTG & 478 \\
\hline
\end{tabular}

Primers for NPHP2

\begin{tabular}{|c|c|c|c|c|c|}
\hline exon & F primer & $5^{\prime}$-nucleotide sequence $-3^{\prime}$ & Rprimer & $5^{\prime}$-nucleotide sequence $-3^{\prime}$ & $\begin{array}{l}\text { Amprified } \\
\text { fragment } \\
\text { length (bp) }\end{array}$ \\
\hline SUTR & I5Uf1 & TTTCCCATTGGGCTCTCGGCC & I5Ur1 & TGAGTCTGCAGCAGGGGCCAA & 366 \\
\hline exon1 & IEx1F & CCCACTTGGAACTGATGAGAC & IEx1R & AACAAACTTCCTCAGGACAAAC & 265 \\
\hline exon2 & IEx2F & ATAATAAACAGCGAATATAGTTCCTAC & IEx2R & TGTCCATTGCATAGTTCCAC & 327 \\
\hline exon3 & IEx3F & GTGGAATTACAAGCATTITTCC & IEx3R & AATTCAGGCCTTCTTCCTTG & 411 \\
\hline exon4 & IEx4F & TTGTTACTGTTGTTATTTCGAGAACC & IEX4R & ACTTCTTGGGGGATGAGTCC & 356 \\
\hline exon5 & IEx5F & CACCAAATGTAATTTATTGAGGATTC & IEx5R & AGTGGAAGGGAAGGCACAG & 317 \\
\hline exon6 & IEx6F & CTGCTGTTCAGAAACCGTTG & IEx6R & GGTGTAGGAGTGCAAAAAGC & 421 \\
\hline exon7 & IEx7F & AGGGGAAAATGCTTTGCTTC & IEx7R & AATTTTATAGCAACATCTACACTTTGG & 351 \\
\hline exon8 & IEx8F & GATGGGGAAATCAAGAGAGG & IEx8R & TGTGCAGCTTTCTGCTAAGG & 348 \\
\hline exon9 & IEx9F & CCATAAGAATAAAGCATTAAAGGAAC & IEx9R & TGTGGGTGATCTCTTCATCTTG & 494 \\
\hline exon10 & IEx10F & CCACATATCCAAAAATACTTACTCC & IEx10R & AGAAAGGATGTATGATAAAGAGCAC & 528 \\
\hline exon11 & IEx11F & TTCCACATCTTAGAATGAAGTTTCC & IEx11R & CTCATCTGTTTCCCTCTCCTG & 427 \\
\hline exon12 & IEx12F & CACACAGAGACTTGAGGAGGTG & IEx12R & CGGCAGAAGATGACAAAGG & 382 \\
\hline exon13 & IEx13F & TGTAACTGCCACTATTATGGTGATG & IEx13R & CACCACATGGAACTCACTGG & 939 \\
\hline exon14 & IEx14F & AATGGGAGCTTGAATGAACC & IEx14R & TGGTACTCTGGGGGTACTTG & 410 \\
\hline exon15 & IEx15F & CACACACCTGCAAGCTCAAG & IEx15R & TCTITGGGGATGAAACAAAGG & 255 \\
\hline exon16 & IEx16F & CCAATGAACTATTCCCTTCAGC & IEx16R & GCAGAAAATCTGAACTCTGCAC & 242 \\
\hline
\end{tabular}

Fig. 1 Genomic DNA extraction, PCR, and determination of $N P H P 1,2,3$ and 4 gene sequence. PCR primers were prepared to amplify approximately 200-300 bp fragments based on NPHP $1-4$ gene sequences registered in GenBank, the following primers were used as shown

has been shown to carry out signal transmission between renal tubular epithelial cells, in cooperation with nephrocystin-1 [3].

NPHP2, the gene responsible for infantile NPH (NPH2), is located on 9q22-31 [4]. NPHP2 encodes a protein termed inversin (INVS). An abnormality in INVS can cause situs inversus, pancreatic islet-cell dysplasia, cardiovascular abnormalities, and hepato-biliary disorders. In addition, INVS abnormalities can cause cyst formation resembling that in juvenile nephronophthisis. However, the renal prognosis is worse progression to ESRD in early childhood.

The gene responsible for adolescent NPH (NPH3), NPHP3 is located on chromosome $3 \mathrm{q} 21-22$ [5]. NPHP3 is believed to encode a protein involved in signal 
Primers for NPHP3

\begin{tabular}{|c|c|c|c|c|c|}
\hline exon & F primer & $5^{\prime}$-nucleotide sequence $-3^{\prime}$ & Rprimer & $5^{\prime}$-nucleotide sequence $-3^{\prime}$ & $\begin{array}{l}\text { Amprified } \\
\text { fragment } \\
\text { length }(b p)\end{array}$ \\
\hline exon1 & NPHP4E01F010 & ATGCAAATCAGGATGGGCCCG & NPHP4E01R010 & AACCCACGTAGCCAAACGGCA & 598 \\
\hline exon2 & NPHP4E02F010 & AGGITCCTCTGGGATTIAGTG & NPHP4E02R010 & AATCAAAGCATCGTAAGCCAG & 373 \\
\hline exon3 & NPHP4E03F010 & TGATATCTGAGCGAGGTGGCC & NPHP4E03R010 & AAGTCTGAGACGCGCTGTGAG & 368 \\
\hline exon4 & NPHP4E04F010 & TGCTGTGGCACGTGTAGGAAG & NPHP4E04R010 & ACTGCACTCTAGCCTGGTTGA & 379 \\
\hline exon5 & NPHP4E05F010 & AAAGCTCTAGTGGGCGTGGTG & NPHP4E05R010 & CAGATAGCAGITTACACTGAG & 273 \\
\hline exon6 & NPHP4E06F010 & CCTGTTIGTGGTGTTICTAAC & NPHP4E06R010 & ПTССАTСCTCCTCСACTGTCC & 426 \\
\hline exon7 & NPHP4E07F030 & TGGAGGAGGTITGGGGTAGAT & NPHP4E07R020 & AGGGGAAAAGACAGAACTACA & 569 \\
\hline exon8 & NPHP4E08F010 & CTGCTCCAGTTTCTTCTCTCT & NPHP4E08R010 & TCCCACGTGGGTGAGTCAACA & 383 \\
\hline exong & NPHP4EO9F010 & ACTTGCTGTTGCAGCAGCACC & NPHP4E09R010 & CCATCTCATCTGTATCCTTTG & 446 \\
\hline exon10 & NPHP4E10F010 & CACTGAGCTCTCGITGAATTT & NPHP4E10R010 & GGCATACCCATGACATGAAAA & 420 \\
\hline exon11 & NPHP4E11F010 & GACTITGITITAGGGCAGAGC & NPHP4E11R010 & ATGTGGTGATTACCGTACTAG & 339 \\
\hline exon12 & NPHP4E12F010 & AGACAAGGTGGTGAGGCCTGT & NPHP4E12R010 & AAGCACGCAGGGATCCACTGT & 274 \\
\hline exon13 & NPHP4E13F010 & TIGAGAAGCGGTCCCAGGTTC & NPHP4E13R010 & TGCCACCTAACTAAGGACAGG & 384 \\
\hline exon14 & NPHP4E14F010 & CCAGAGGCAATTAATCGATGA & NPHP4E14R010 & ATTGATGCACCTCCCTGTGGA & 354 \\
\hline exon15 & NPHP4E15F010 & CAGACTGTTGGACCTTGTGAA & NPHP4E15R010 & TCAGCACAGACAGTTCTGCCA & 392 \\
\hline exon16 & NPHP4E16F010 & GACTAAGGTGCCTGGACCATC & NPHP4E16R010 & GGTCACCGTATGATTCTAATG & 419 \\
\hline exon17 & NPHP4E17F010 & GTAGCTATGACAGAAGCAGAA & NPHP4E17R010 & ACAAGTCCTGTGCGGGATAGC & 392 \\
\hline exon18 & NPHP4E18F010 & AGGGTCTTATCCTGCGCACAC & NPHP4E18R010 & ATTCTCCCGGTTTCCTCCTGG & 441 \\
\hline exon19 & NPHP4E19F010 & AGGCCATTTGAAAGCCACAGC & NPHP4E19R010 & CACATGCACACACGCATGCAC & 326 \\
\hline exon 20 & NPHP4E2OF010 & CССTCCCTATATAGGTGGTCC & NPHP4E2OR010 & AGGTAAGAGAGAATCATGTGG & 404 \\
\hline exon21 & NPHP4E21F010 & AATGTCCTCTTCTGAGATCGC & NPHP4E21R010 & AGAGAAGTCAAATCGCCCCGG & 444 \\
\hline exon22 & NPHP4E22F010 & TCTCTCCCACTCCTCTGAGCA & NPHP4E22R010 & TGCACAGGTAAGGGAGGGACA & 391 \\
\hline exon 23 & NPHP4E23F010 & TCAGTGTGAGAGGAGGCTGGT & NPHP4E23R010 & AAAAGGCCATTCCCAGGCCCA & 346 \\
\hline exon24 & NPHP4E24F010 & GTCTTGGCAACAGTGGAGATA & NPHP4E24R010 & ACCAGGGCATGAAGCCATGAG & 360 \\
\hline exon 25 & NPHP4E25F010 & TGACGAGCCTGTCCTGTCCTA & NPHP4E25R010 & CCTAAAATGAAGAGGATCCCA & 286 \\
\hline exon26 & NPHP4E26F010 & AGATGCGITCTGGGAGGGACT & NPHP4E26R010 & TITAGGAAGGGGCCAAGCCCA & 308 \\
\hline exon27 & NPHP4E27F010 & TITCCCTGCACAGCCTCCTGT & NPHP4E27R010 & AAAAGCTGCTGTCAGGGCCAC & 390 \\
\hline exon28 & NPHP4E28F010 & AACCACCCATGACCTTGGGCT & NPHP4E28R010 & TGTATCCAGTGGTCCGAGTCA & 392 \\
\hline exon29 & NPHP4E29F010 & TCTTATCTCTGTGGGGGTCCC & NPHP4E29R010 & GCTGGTGATTTGAGGAACTCG & 364 \\
\hline exon30 & NPHP4E3OF010 & CAGCTCCCCTTGGAAATAAAC & NPHP4E30R020 & AAACTGCCAAGGGAAGACGTG & 768 \\
\hline
\end{tabular}

Primers for NPHP4

\begin{tabular}{|c|c|c|c|c|c|}
\hline exon & F primer & $5^{\prime}$-nucleotide sequence $-3^{\prime}$ & Rprimer & $5^{\prime}$-nucleotide sequence $-3^{\prime}$ & $\begin{array}{l}\text { Amprified } \\
\text { fragment } \\
\text { length }(b p)\end{array}$ \\
\hline exon1 & NPHP3E01F020 & TGCTCCGCCCAGTCCTGCTCT & NPHP3E01R020 & GAGAATATGGCCTCTCAAATT & 694 \\
\hline exon2 & NPHP3E02F010 & CATGAAGTTCCTGATAATTGG & NPHP3E02R010 & GAATCCTACATGACTTACTTC & 387 \\
\hline exon3 & NPHP3E03F010 & GAGGACCAAATGAATATTGGT & NPHP3E03R020 & GCAGCTGACAGAGAGAACACA & 420 \\
\hline exon4 & NPHP3E04F020 & CAGTATCTITGAACTTTGCCA & NPHP3E04R020 & GATGGTITGTCAATGGAAAGC & 459 \\
\hline exon5 & NPHP3E05F020 & GGTATGGCAGTATTAACATGT & NPHP3E05R020 & GCTTCCTGTTCTTTAAGACAT & 391 \\
\hline exon6 & NPHP3E06F020 & GTATTTGAGAGAAACTTGCCT & NPHP3E06R020 & GCTATATTTGCCAAACTCTGA & 595 \\
\hline exon7 & NPHP3E07F020 & GITGGACCTITTCTGGCCACT & NPHP3E07R020 & GTTCCAGCCACACTGGTTTCT & 401 \\
\hline exon8 & NPHP3E08F010 & CCTAAGGTTGTTGTGAAGATA & NPHP3E08R010 & TICAAAAAGACAAGCAAGTGG & 320 \\
\hline exong & NPHP3E09F020 & AAGGCTGTATGTITGAACTTG & NPHP3E09R020 & CACATCTCAACATGGATAATC & 440 \\
\hline exon10 & NPHP3E10F010 & CAGCTTITCTCCAGTATTTTC & NPHP3E10R020 & GGGCATGAACCTATTGTTTTA & 350 \\
\hline exon11 & NPHP3E11F020 & AGTAACTGACCACCTGATTGC & NPHP3E11R020 & GACCCGATTGTATCGAATATT & 390 \\
\hline exon12 & NPHP3E12F020 & ATATTCGATACAATCGGGTCC & NPHP3E12R020 & CTGTGGGCATACGATATATTT & 458 \\
\hline exon13 & NPHP3E13F010 & CAGAGTTCAGATTGGTGATAA & NPHP3E13R010 & CCTCACTGCAAGTTACATAAA & 406 \\
\hline exon14 & NPHP3E14F010 & GTTGTGATTCATTGCTCAAAG & NPHP3E14R010 & CCTTATAACAGATCCCCTATA & 410 \\
\hline exon15 & NPHP3E15F010 & TITCTGTCGGGGTACTTGTTG & NPHP3E15R010 & CAGACTGGTGTAGTGATCAGT & 283 \\
\hline exon16 & NPHP3E16F020 & TGACTCTAGCAGCCCCATAAA & NPHP3E16R020 & GGCTATCAGCATTCCTGCATA & 435 \\
\hline exon17 & NPHP3E17F020 & GTTATCTITGGTGTGCTAGAT & NPHP3E17R010 & CTITGGCAGAAATAATCTTGC & 487 \\
\hline exon18 & NPHP3E18F010 & CATTCCACACTTCTGAGATTT & NPHP3E18R010 & GAATAGGGAGAGGATTTAATC & 496 \\
\hline exon19 & NPHP3E19F020 & GGTTCTGCATATCACTGAATT & NPHP3E19R020 & GGAAAAGCAGATCTAATAGAG & 492 \\
\hline exon 20 & NPHP3E2OF010 & CAGTACTCGCCTACTAATAAA & NPHP3E20R020 & GCAAGATCTGTCATCTGATTA & 440 \\
\hline exon21 & NPHP3E21F020 & СTCTTCTCTTTTTCCAAGATG & NPHP3E21R020 & CCACATGAAGACTAGGCACAG & 497 \\
\hline exon22 & NPHP3E22F020 & CTAGACTTGCTITGTITTGTC & NPHP3E22R020 & CTITAAAGAACTGAGGTAGCT & 614 \\
\hline exon23 & NPHP3E23F010 & GTTGCCATGTGGAAATATTTG & NPHP3E23R010 & CATACATGAAATTITGCGTGG & 436 \\
\hline exon24 & NPHP3E24F010 & GGAAAGTAAGATTITGAGCTG & NPHP3E24R020 & GTTCTGCTCAGTTACTTGTTA & 536 \\
\hline exon 25 & NPHP3E25F020 & GCTITICTATACAGTGTAGCT & NPHP3E25R010 & ССTTCATACAAGTCTAACTTC & 485 \\
\hline exon26 & NPHP3E26F010 & CCCATCTITTAGGAGGATATT & NPHP3E26R010 & CCCCACTCTAAGAAAAAACAT & 341 \\
\hline exon 27 & NPHP3E27F010 & AGGGGAAATGGGCAAATATTI & NPHP3E27R020 & CCTTGGATACCATATAATAGG & 512 \\
\hline
\end{tabular}

Fig. 1 continued 
Fig. 2 Percentage of NPH patients with $N P H P$ gene mutation. NPHP gene mutation was detected in 19 patients. No NPHP gene aberration detected within the sequences analyzed in the other 16 patients with suspicion of NPH clinicopathologically

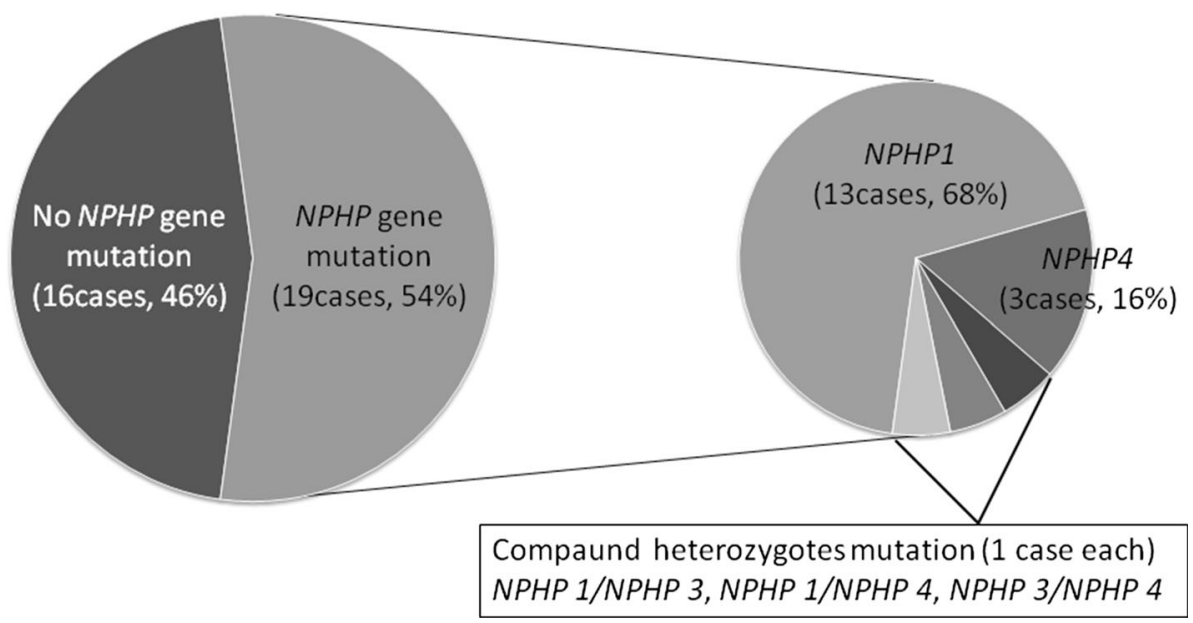

transmission in renal tubular epithelial cells, such as signaling involving diacylglycerol kinase-zeta and receptorlike tyrosine kinase. Abnormalities of the protein disrupt urinary concentrating ability and the structure of cilia of renal tubules, as in the other types of NPH.

Previous reports describe occurrence of NPHP1 mutations in approximately 30-50 \% of juvenile nephronophthisis patients in Western countries [1, 6], where genetic analysis of NPHP1 is performed initially when juvenile $\mathrm{NPH}$ is suspected. If mutation is detected, kidney biopsy usually is deferred [7]. Genetic diagnosis is made less frequently in Japan; so kidney biopsy often is performed to obtain a definitive diagnosis. Not infrequently, NPH is discovered in the advanced or end stage in many Japanese patients, in whom treatment no longer can slow progression. Unfortunately, symptoms typically seen in early stages are incompletely characterized.

In the present study, we investigated clinical, histologic, and genetic features in 35 Japanese patients clinically and histologically suspected to have NPH, aiming to promote early diagnosis. We studied many exons as many as 13 NPHP genes. Since such genetic analysis involves significant cost and time, we also screened biopsy specimens by immunohistologic methods employing antibodies against relevant peptides.

\section{Methods}

\section{Patient registration and informed consent}

Our subjects included 35 patients with clinicopathologic findings suggestive of NPH who were referred to our department from various regions of Japan. The study was performed following approval by the Ethics Committee of Kinki University Faculty of Medicine and acquisition of written informed consent from patients or their parents (Actual state of Japanese juvenile nephronophthisis patients and identification of gene aberrations; approval number 20-99).

\section{Genomic DNA extraction, polymerase-chain reaction (PCR), and determination of $\mathrm{NPHP}$ gene sequence.}

After approximately $5 \mathrm{~mL}$ of peripheral blood was collected from patients into tubes containing Na-EDTA, genomic DNA was extracted using NucleoSpin for Blood (TaKaRa Bio Inc, Shiga, Japan). Human genomic DNA (TaKaRa Clontech, code 636401; Shiga, Japan) was used as a control. Patient samples and control genomic DNA were diluted with sterile water to prepare $10 \mathrm{ng} / \mu \mathrm{L}$ solutions. PCR was performed using these as templates and TaKaRa PCR Thermal Cycler Dice Gradient (TaKaRa Bio Inc, Shiga, Japan). To determine extent of deletions and identify break points, PCR primers were prepared to amplify approximately 200-300 bp fragments based on NPHP gene sequences registered in GenBank (Fig. 1). For PCR, annealing temperatures and times were $63{ }^{\circ} \mathrm{C}$ and $15 \mathrm{~s}$ for $N P H P 1$ and NPHP3; $60{ }^{\circ} \mathrm{C}$ and $15 \mathrm{~s}$ for NPHP2; and $60{ }^{\circ} \mathrm{C}$ and $20 \mathrm{~s}$ for $N P H P 4$, respectively. For sequence analysis, PCR products were purified by an enzyme reaction, and templates for sequencing were prepared. The sequencing reaction was carried out using the prepared template DNA and a BigDye Terminator v.3.1 Cycle Sequencing Kit (Applied Biosystems, CA, USA), employing the dye terminator method. Reaction products were purified by gel filtration, and sequence analysis was performed using a capillary-type sequencer, ABI3730x1 (Applied Biosystems, CA, USA). The algorithm established by Salomon et al. [8]. was adapted for use in our analytical procedure. In children with renal dysfunction 


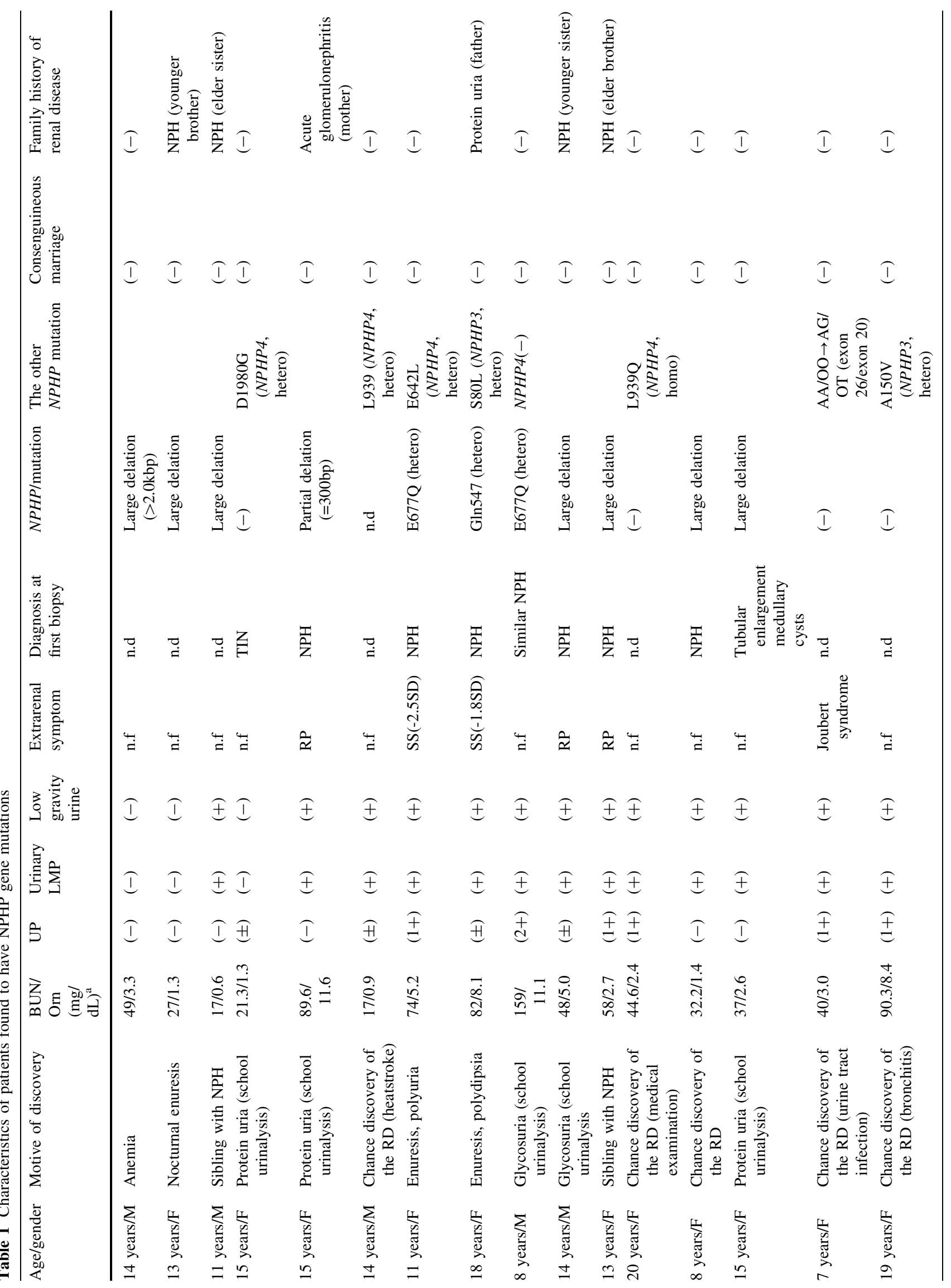




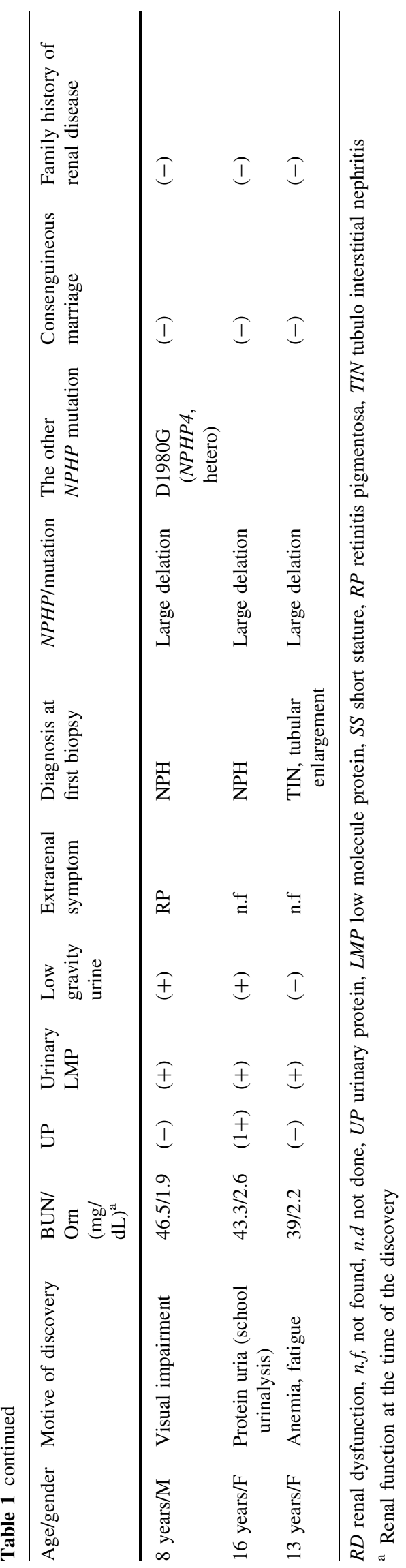

who were 5 years old or younger, the gene responsible for infantile NPHP (NPHP2) was analyzed first. In patients older than 5 years, NPHPI was analyzed first; if no mutation was detected, NPHP4 was examined. NPHP3 analysis was added when no mutation was detected in other genes in patients whose disease progressed to endstage renal disease at an age of 16 years or older.

\section{Clinical data}

Data originally collected at our department as well as data provided by other institutions were surveyed using a questionnaire. Questionnaire consists of personal data including the patient's age, motive of discovery, urinary abnormality and renal dysfunction, detailed clinical data, extrarenal symptom, renal tissue diagnosis at the first biopsy, consanguineous marriage, and family history of renal disease.

\section{Results}

\section{NPHP gene analysis}

Among 35 patients, an NPHP gene mutation was identified in 19 patients. Although NPH was suspected clinicopathologically in the other 16 patients, no NPHP gene aberration was detected within the sequences analyzed (Fig. 2). Characteristics of patients with NPHP1 gene mutations (Table 1) and without NPHP gene mutations (Table 2) were shown. A mutation was detected only in NPHP1 in 13 patients; deletion was extensive in 10 (Fig. 3a) and partial in 1. Two other patients had a point mutation (E677Q and K334 N, both heterozygous). In all, these mutations accounted for $37.1 \%$ (13/35) of patients. In another candidate gene responsible for the juvenile type, NPHP4, the mutation L939* was detected in 2 patients (Fig. 3b), while a D1980G mutation was detected in 1 , accounting for $8.6 \%$ (3/35) of all patients. Compound heterozygotes containing 1 mutation each in NPHP1 G547* and NPHP3 S80L (Fig. 4a), 1 mutation each in NPHP1 E677Q and NPHP4 E642L (Fig. 4b), and 1 mutation each in NPHP3 A150 V and NPHP4 D1089G (Fig. 4c) also were observed. The disease progressed to ESRD before 20 years of age in these patients, similar to the course of other patients with a single-gene mutation. No NPHP2 mutation was detected in any patient.

\section{Clinical and demographic features of patients}

\section{Patient background}

Patients were reported from 46 prefectures without evident selection bias, and with no important regional 


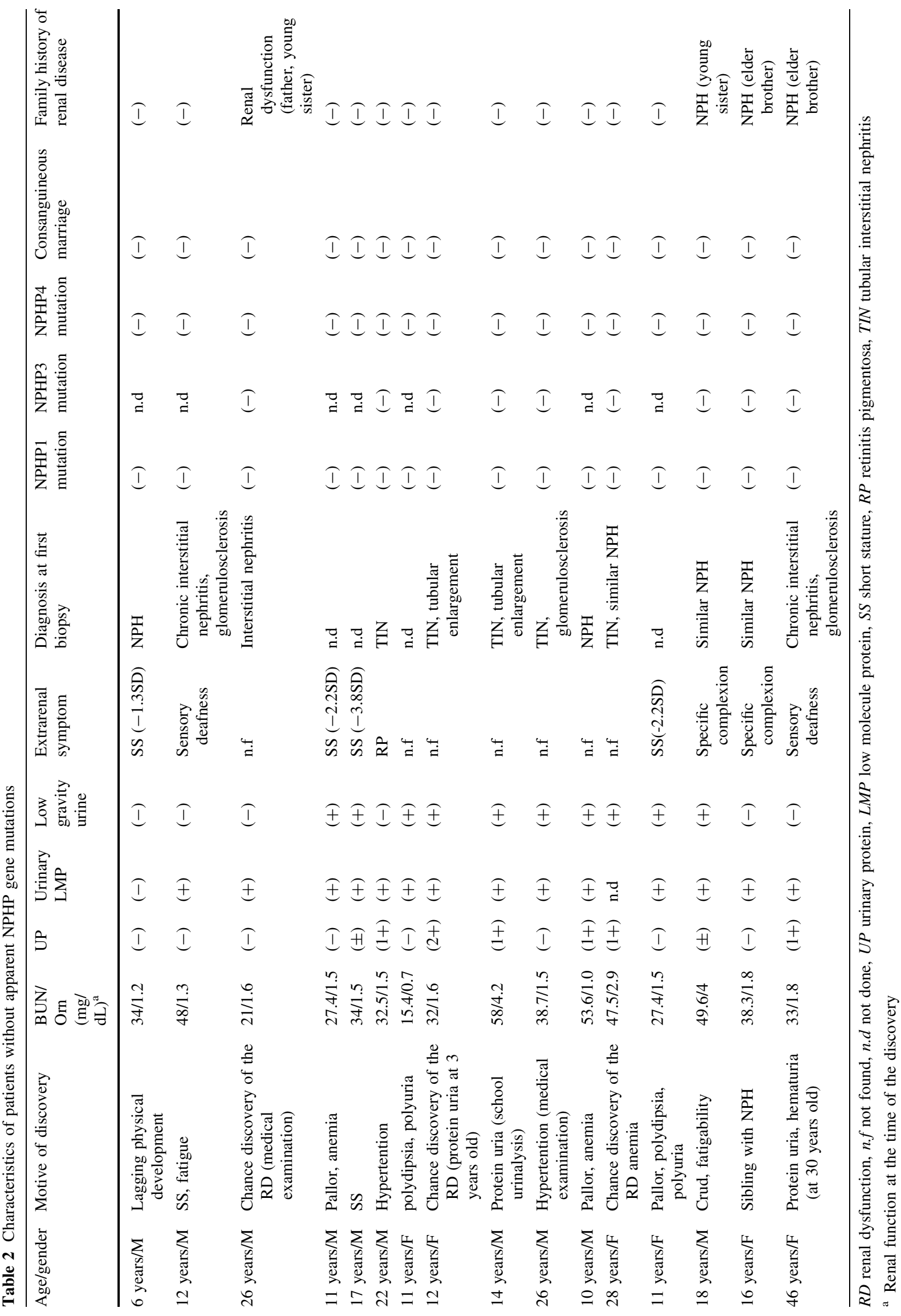


(a)

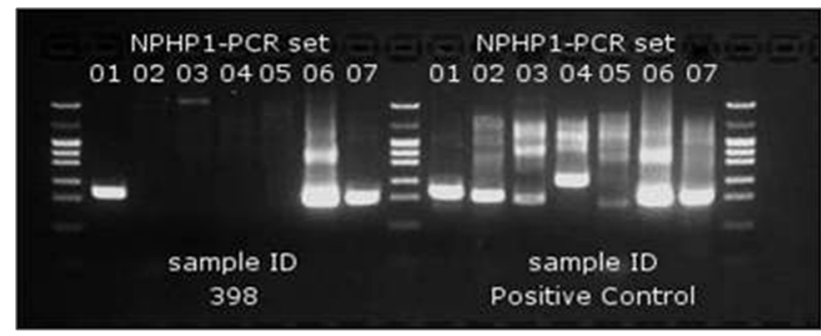

(b)

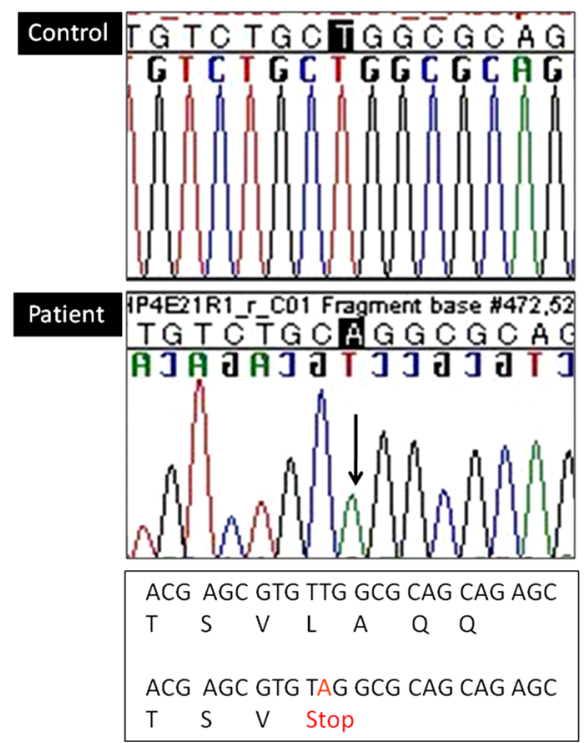

Fig. 3 Analysis of deletion in NPHP1 (a) and analysis of NPHP4 (b). In a lane 1 and lanes 6 and 7, contain PCR products of regions within and outside NPHP1, respectively; Lane 2 contains PCR products from the junction between NPHP1 and the adjacent $M A L L$ gene. Lanes 3-5 show the PCR products of NPHP1 obtained with primers amplifying fragments of approximately $300 \mathrm{bp}$. NPHP1 was nearly completely deleted (1.2 kbp deletion). In $\mathbf{b}$, substitution of TAG for TTG formed a stop codon, prematurely terminating peptide synthesis

differences (Fig. 5). The male:female ratio was 16:19, with evident gender difference. Ages of patients ranged from 2 to 38 years (median; 12.5). Familial occurrence was noted in 3 families. Other occurrences were solitary, with no family member showing a urinary abnormality, a diagnosis of NPH, or any renal dysfunction of unknown cause.

\section{Initial abnormality deletion}

NPH sometimes was discovered following an abnormal urinary finding by mass screening, such as proteinuria detected in a urine test at school $(18 \%)$, or renal dysfunction discovered incidentally in working up other medical symptoms, or during medical check-ups $(23 \%)$. Approximately $20 \%$ of cases were discovered because of urinary tract symptoms such as polyuria with or without polydipsia, enuresis (often nocturnal), or mellituria. Some $38 \%$ were discovered because of either extrarenal manifestations such as lagging physical development, dwarfism, anemia, pallor, hypertension, or visual disturbance arising from pigmentary retinal degeneration; a prior diagnosis of NPH in a sibling; or both (Fig. 6).

\section{Urinary findings}

Urine specific gravity frequently was low (not greater than 1.010); approximately $75 \%$ of cases. Low molecular weight proteinuria, such as $\beta 2$-microgloburinuria, also was common $(85 \%)$, even though inclusion of renal function shown such as between blood urea nitrogen and serum creatinine was relatively mild at that time.

\section{Renal histologic findings}

Renal biopsy was performed in 25 patients (71\%). These included 13 patients demonstrated to have an NPHP gene mutation and in 12 with no NPHP gene mutation identified (suspected cases). Histologic findings included suspected NPH; interstitial nephritis, renal tubular dilation, and glomerulosclerosis. Cystic dilation of renal tubules and irregular contours of tubular basement membranes were observed in most patients, mainly in the renal medulla (Fig. 7a). Sclerotic glomeruli, inflammatory cell infiltration in the renal tubules and interstitium, and fibrosis were frequent, although not seen in all patients (Fig. 7b).

\section{Discussion}

Renal tubular epithelial cells are attached to the basement membrane through integrin cross-linking, which transmits extracellular signals to the cell nucleus [2]. Nephrocystin acts importantly in signal transmission between tubular epithelial cells and between these epithelial cells and the extracellular matrix functioning as a docking protein. Nephrocystin also is involved in cell adhesion, together with $N$-cadherin, catenin, and $\beta$-catenin $[2,8]$. Furthermore, nephrocystin influences actin cytoskeleton structure together with $\beta$-tubulin, contributing to maintenance of the cytoskeleton and determination of cell polarity. Nephrocystin forms a complex with Crk-associated substrate, which promotes phosphorylation of Pyk2 and transmits intracellular information through a Pyk2-dependent pathway [2]. Furthermore, nephrocystin is present on primary cilia, where it functions in cooperation with $\alpha$-tubulin; nephrocystin also is involved in signal transmission in organelles [9]. Accordingly, abnormalities in the nephrocystin molecule disrupt signal transmission between cells 
Fig. 4 Compound heterozygotes with heterozygous mutations in different NPHP genes. In a, a compound heterozygote has one heterozygous mutation involving each of NPHP1 (G547*) and NPHP3 (S80L). In b, a compound heterozygote has one heterozygous mutation involving each of $N P H P 1$ (E677Q) and NPHP4 (E642L). In $\mathbf{c}$, a compound heterozygote has one heterozygous mutation involving each of NPHP3 $(\mathrm{A} 150 \mathrm{~V})$ and NPHP4 (D1089G)

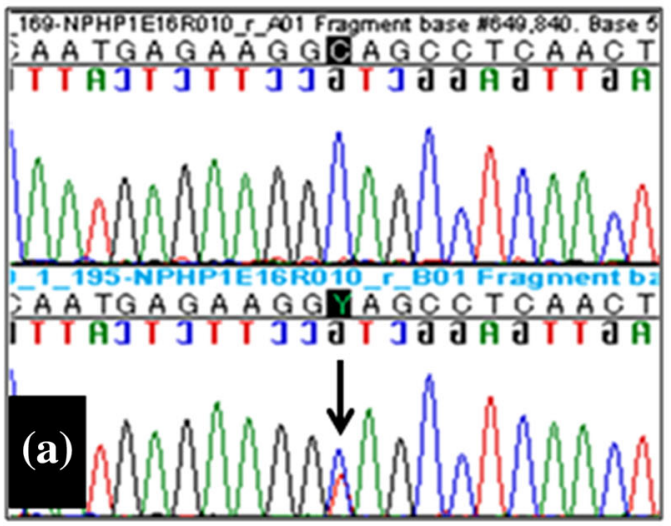

NPHP1 (exon20: E677Q)

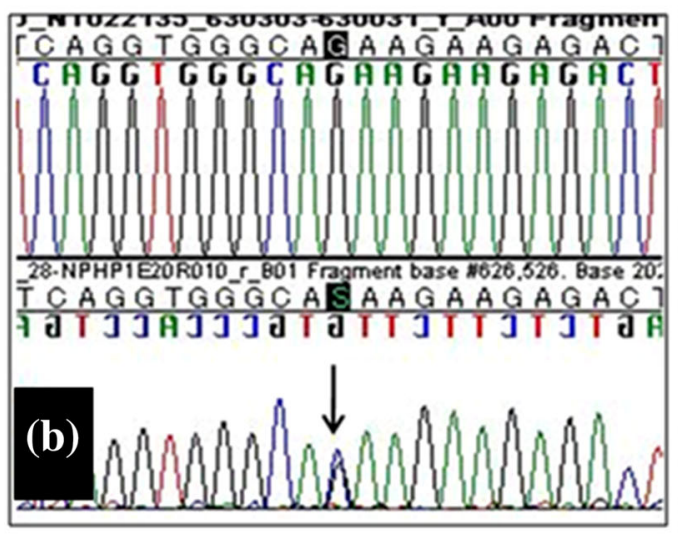

NPHP3 (exon2:A150V)

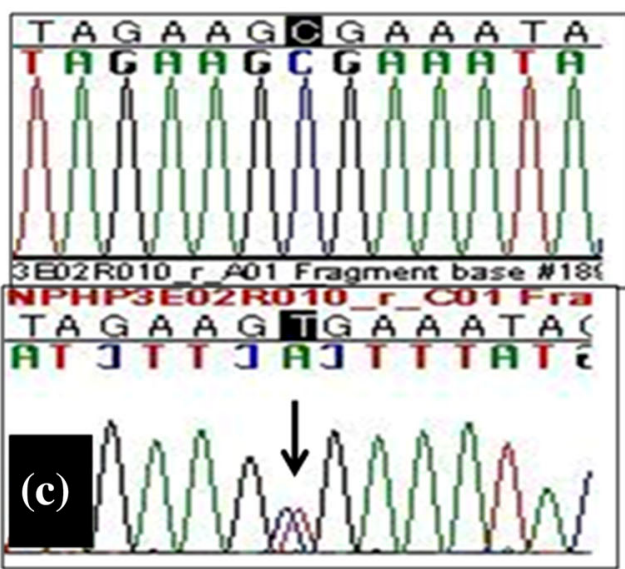

NPHP3 (exon1: S80L)

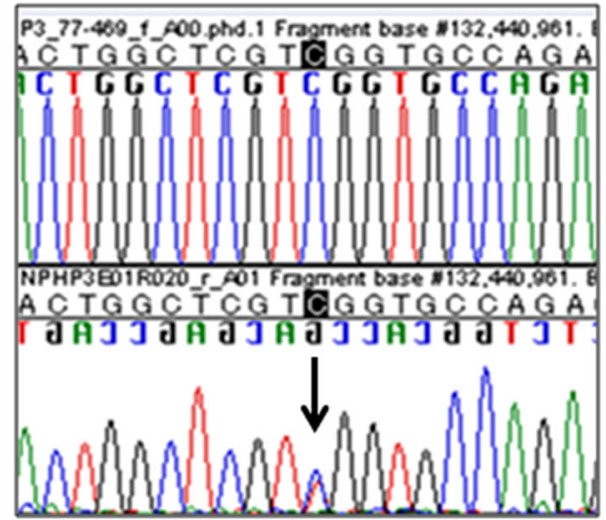

NPHP4 (exon15: E642L)

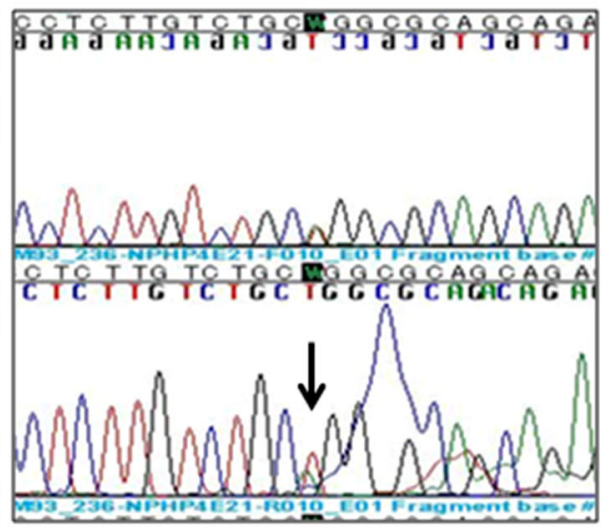

NPHP4 (exon23: D1089G)

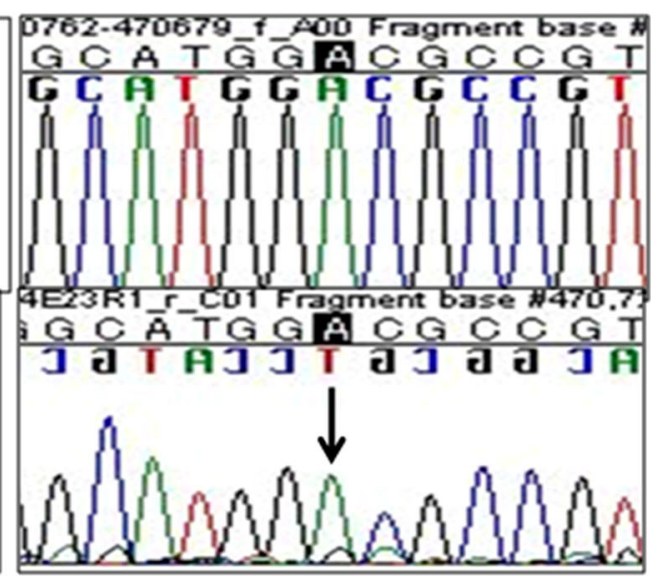

and the extracellular matrix, intercellular adhesion, cytoskeletal integrity, cell polarity, primary cilia function, and intracellular signal transmission to the nucleus. Structural and functional disorders involving the renal tubular epithelium result.

An NPHP gene mutation was detected in about $54 \%$ of all patients, but no mutation was noted within the sequences analyzed in the other $46 \%$. However, nephronophthisis was suspected clinically and histologically, suggesting possible mutation in some other NPHP gene. An NPHPl mutation was most frequent among our Japanese patients, most often representing a large deletion rather than a point mutation. Frequency of an NPHP1 mutation was similar to that reported in Western populations [10].

On the other hand, mutation in the gene responsible for the infantile type, $N P H P 2$, a patient in a compound heterozygous stable with another abnormal NPHP gene such as that responsible for NPH3 recently has been 


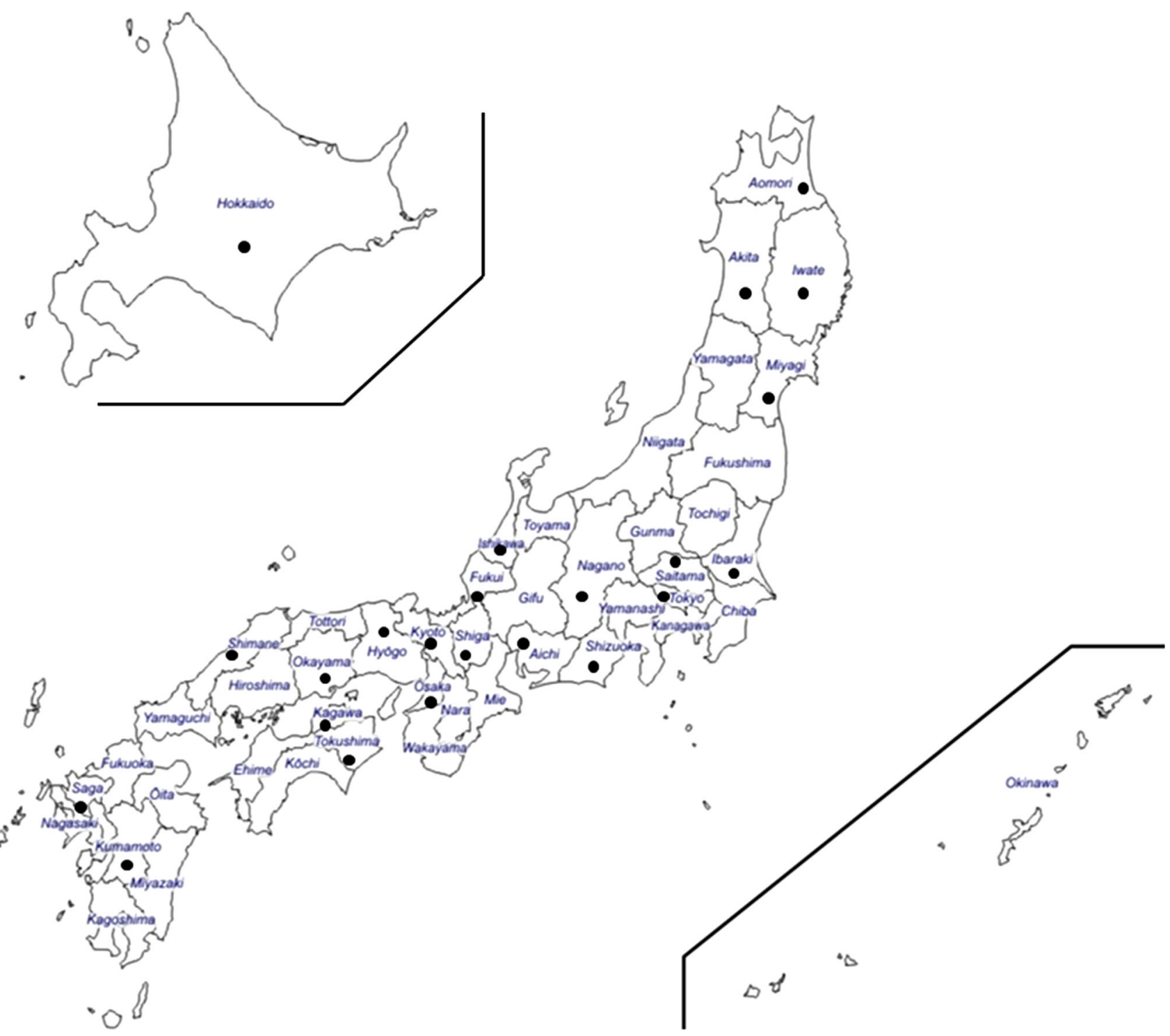

Fig. 5 Demographic features of patients in Japan. Regional distribution of study subjects within Japan, show as a dot for each patient

reported [11, 12]. We also found compound heterozygosity across multiple NPHP genes in some of our Japanese nephronophthisis patients. In NPHP4, L939* (IVS$20 \mathrm{~T}>\mathrm{A}$ ) was detected in two geographically distant patients who were not consanguineous. The mutation formed a stop codon by substituting TAG for TTG in exon 21 , terminating peptide synthesis. This might prove to be a 'hot spot' among Japanese patients.

We detected in three patients with two mutations in either NPHP1, NPHP3, or NPHP4 in this study. As similar to the results of the other studies $[13,14]$, the age of the initial discovery of this disease and the course of progression to end-stage renal disease were not significantly different from those of the patients having mutation in single NPHP gene. An analysis of patient backgrounds revealed that NPH was distributed fairly evenly Japan, including the suspected cases where no causative mutation was identified. Heterozygotes carrying NPHP gene mutations also were rather evenly distributed nationwide. No gender difference was evident from our analysis. Although the median age at time of disease discovery was 12.5 years, individual presentation ranged from infantry to adulthood.

Frequency of disease discovery in mass screening programs, such as school urine tests, was low, as previously reported [15]. Incidental discovery of renal dysfunction during diagnostic workup of possibly unrelated symptoms, or during routine check-ups, accounted for less than $50 \%$ of cases. Often symptoms that led to the discovery of NPH represented extrarenal manifestations such as incomplete physical development reported previously [15]. In particular, currently used urine test strips, intended mainly to detect albuminuria, are insensitive to this disease. 


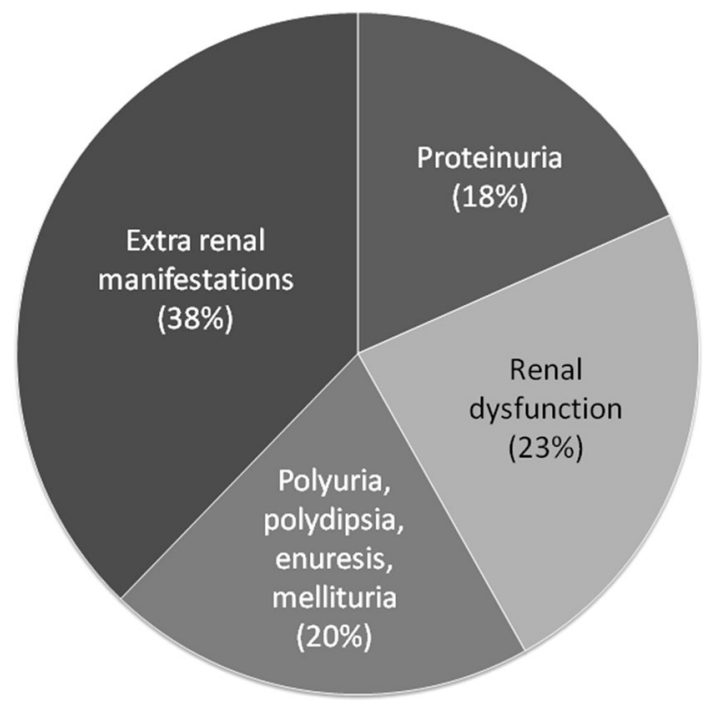

Fig. 6 Clinical suspicion and motivation to discover for NPH. Proteinuria is detected in a urine test at school (18\%), renal dysfunction discovered incidentally (23\%), urinary tract symptoms such as polyuria with or without polydipsia, enuresis, or mellituria (approximately $20 \%$ ). Some $38 \%$ were discovered because of either extrarenal manifestations such as lagging physical development, dwarfism, anemia, pallor, hypertension, or visual disturbance arising from pigmentary retinal degeneration

Development in siblings was noted in three families, suggesting autosomal recessive inheritance. However, many cases appear to be sporadic. Familial genetic analysis centering on patients, parents is needed.

In contract to albuminuria, urinary findings such as low specific gravity and low-molecular-weight proteinuria are relatively helpful in early discovery. According to the results of this study, we suggest that the findings of the low-molecular weight proteinuria and hypotonic urine reflecting renal tubular disorder coupled with the histologic abnormalities involving cystic dilation of renal tubules and the irregularity of tubular basement membrane could be a convincing diagnostic criterion of this disease. Extrarenal manifestations, such as short stature, delayed physical development, and anemia also were frequent. Unfortunately, these tended to coincide with were progression of renal dysfunction rather than early NPH. Nonetheless, NPH needs to be considered in children with such presentations. Some patients have been reported to show somewhat distinctive extrarenal manifestations [13] such as pigmentary retinal degeneration (Senior-Loken syndrome), ocular dysmetria (Cogan's syndrome), cerebral ataxia, hepatic fibrosis, and skeletal and facial abnormalities [13, 16, 17]. Even the most frequent of these extrarenal manifestations, pigmentary retinal degeneration, was present only in some patients and not in others, even among children showing the same NPHP1 deletion. Similar lesions also have been reported in Jeune, Joubert, oro-facial-digital (OFD1), and
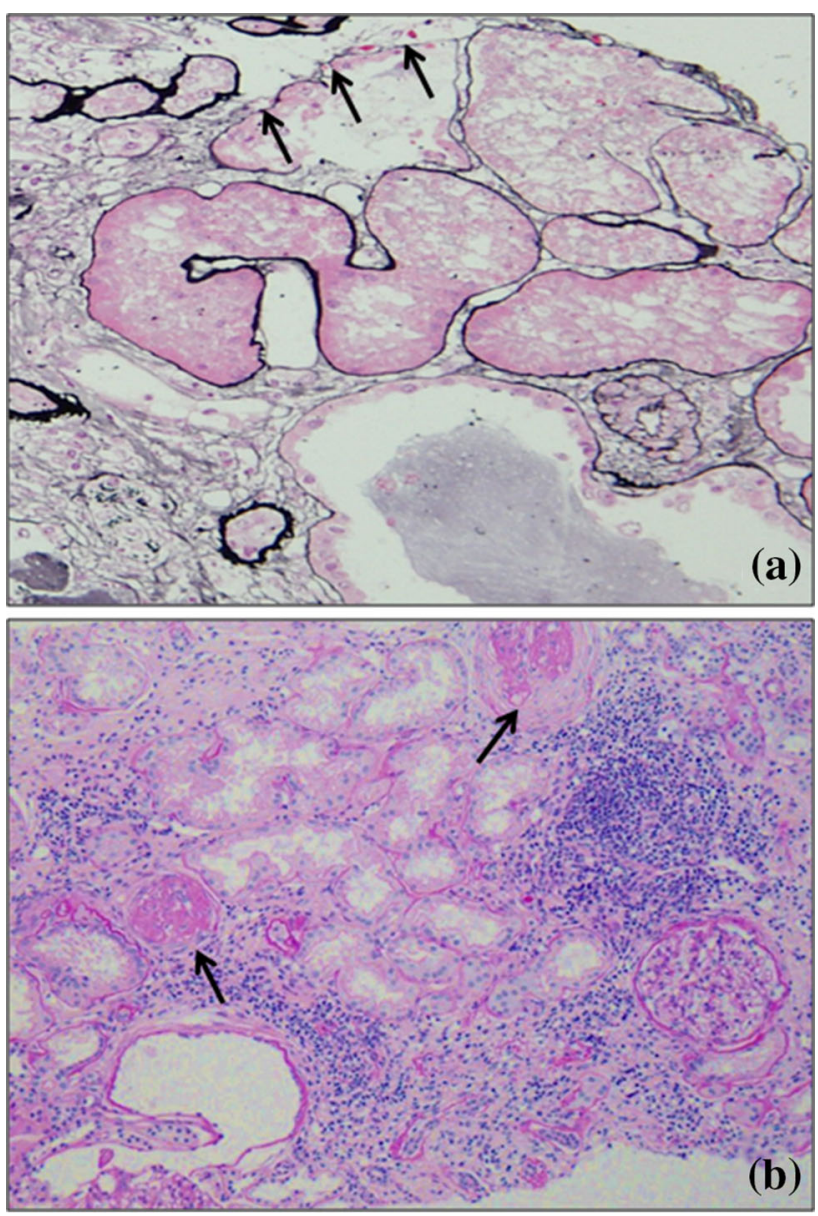

Fig. 7 Pathologic findings in the kidney in nephronophthisis patients. In a irregularity (arrow) of the renal tubular basement membrane was evident (methenamine silver stain, $\times 200$ ). In $\mathbf{b}$, Inflammatory cell infiltration involved the renal tubular interstitium, and sclerotic glomeruli (arrow) were present (periodic acid-Schiff stain, $\times 100$ )

Meckel syndromes [13, 18, 19]. NPHP1 mRNA is expressed predominantly in a wide range of extrarenal tissues including pituitary gland, spine, testis, lymph nodes, and thyroid [14]. Expression also is high in the central nervous system, which could account for associated cerebellar ataxia. However, associated symptoms may develop in organs with low NPHPl expression, such as hepatic fibrosis. The role of nephrocystin in extrarenal manifestations remains poorly understood. The $11 \mathrm{~kb}$ interval between the $3^{\prime}$ end of NPHPI and an inverted repeat containing the distal deletion breakpoint was found to contain the first exon of a second gene, MALL [20]. Although the detail of the MALL gene function has not been clarified, recent report suggested the involvement of the age-related macular degeneration (AMD) [21]. Interestingly, associations have also been reported between AMD and chronic kidney disease [22]. Since pigmentary retinal degeneration is the most common extrarenal 
manifestation of NPH, similar to AMD, MALL gene may involve the pathogenesis of this eye disorder found in NPH patients as the contiguous gene syndrome.

No truly effective treatment currently is available for NPH. Dietary therapy and administration of ion exchange resins and bicarbonate are carried out to manage hyponatremia, hyperkalemia, or metabolic acidosis. Studies possibly relevant to drug therapy have been conducted in various animals, even protozoa [23, 24]. Previous studies reported that renal cyst expression was inhibited by stimulating the G-protein-coupled calcium sensing receptor and elevating $\mathrm{Ca}^{2+}$ and cAMP in the renal tubular epithelial cells of pcy mice. Morphology and function of cilia in zebrafish with ciliopathy may be improved by the administration of rapamycin and rescovitine [25, 26]; however, applicability to human NPHP is unknown. Living-donor kidney transplantation was found to have favorable outcome in many reports including the North American Pediatric Renal Trials and Collaborative Studies (NAPRTCS) [27].

Acknowledgments This study was performed after approval by the Ethics Committee of Kinki University Faculty of Medicine. Written informed consent was obtained from the patient's guardian for genetic examination. We thank Ai Itoh for technical support in tissue staining and manuscript preparation.

\section{Compliance with ethical standards}

Conflicts of interest This study was partly supported by a Grant-inAid for Scientific Research from Morinaga Hoshikai to Tsukasa Takemura (2013-2014) and from Ministry of Health, Labour and Welfare Japan (grant number: 26070201, Representative investigator: Kazumoto Iijima, Pediatrics, Kobe University School of Medicine). The authors declare that they have no competing interests involving this work.

\section{References}

1. Hildebrandt F, Otto E. Molecular genetics of the nephronophthisis-medullary cystic disease complex. J Am Soc Nephrol. 2000;11:1753-61.

2. Donaldson JC, Dise RS, Ritchie MD, Hanks SK. Nephrocystinconverted domains involved in targeting to epithelial cell-cell functions, interaction with filaments, and establishing cell polarity. J Biol Chem. 2002;277:29028-35.

3. Mollet G, Salomon R, Gribouval O, Silbermann F, Bacq D, Landthaler G, Milford D, Nayir A, Rizzoni G, Antignac C, Saunier $\mathrm{S}$. The gene mutated in juvenile nephronophthisis type 4 encodes a novel protein that interacts with nephrocystin. Nat Genet. 2002;32:300-5.

4. Otto EA, Schermer B, Obara T, O'Toole JF, Hiller KS, Mueller AM, Ruf RG, Hoefele J, Beekmann F, Landau D, Foreman JW, Goodship JA, Strachan T, Kispert A, Wolf MT, Gagnadoux MF, Nivet H, Antignac C, Walz G, Drummond IA, Benzing T, Hildebrandt F. Mutations in INVS encoding inversin cause nephronophthisis type 2, linking renal cystic disease to the function of primary cilia and left-right axis determination. Nat Genet. 2003;34:413-20.
5. Omran H, Fernandez C, Jung M, Häffner K, Fargier B, Villaquiran A, Waldherr R, Gretz N, Brandis M, Rüschendorf F, Reis A, Hildebrandt F. Identification of a new gene locus for adolescent nephronophthisis, on chromosome 3q22 in a large Venezuelan pedigree. Am J Hum Genet. 2000;66:118-27.

6. Broyer M, Kleinknecht C. Structural tubulointerstitial disease: nephronophthisis. In: Morgan SH, Grunfeld JP, editors. Inherited disorders of the kidney. Investigation and management. Oxford: Oxford University Press; 1998. p. 340-8.

7. Hildebrandt F, Rensing C, Betz R, Sommer U, Birnbaum S, Imm A, Omran H, Leioldt M, Otto E. Arbeitsgemeinschaft für Paediatrische Nephrologie (APN) Study Group. Establishing an algorithm for molecular genetic diagnostics in 127 families with juvenile nephronophthisis. Kidney Int. 2001;59:434-45.

8. Salomon R, Saunier S, Niaudet P. Nephronopthisis. Pediatr Nephrol. 2009;24:2333-44.

9. Hurd TW, Hildebrandt F. Mechanisms of nephronophthisis and related ciliopathies. Nephron Exp Nephrol. 2011;118:e9-14.

10. Wolf MT. Nephronophthisis and related syndromes. Curr Opin Pediatr. 2015;27:201-11.

11. Tory K, Rousset-Rouvière C, Gubler MC, Morinière V, Pawtowski A, Becker C, Guyot C, Gié S, Frishberg Y, Nivet H, Deschênes G, Cochat P, Gagnadoux MF, Saunier S, Antignac C, Salomon R. Mutations of NPHP2 and NPHP3 in infantile nephronophthisis. Kidney Int. 2009;75:839-47.

12. Hoefele J, Wolf MT, O'Toole JF, Otto EA, Schultheiss U, Dêschenes G, Attanasio M, Utsch B, Antignac C, Hildebrandt F. Evidence of oligogenic inheritance in nephronophthisis. J Am Soc Nephrol. 2008;18:2789-95.

13. Benzing T, Schermer B. Clinical spectrum and pathogenesis of nephronophthisis. Curr Opin Nephrol Hypertens. 2012;21:272-8.

14. Hildebrandt F, Zhou W. Nephronophthisis-associated ciliopathies. J Am Soc Nephrol. 2007;18:1855-71.

15. Hirano D, Fujinaga S, Ohtomo Y, Nishizaki N, Hara S, Murakami H, Yamaguchi Y, Hattori M, Ida H. Nephronophthisis cannot be detected by urinary screening program. Clin Pediatr (Phila). 2013;52:759-61.

16. Ronquillo CC, Bernstein PS, Baehr W. Senior-Løken syndrome: a syndromic form of retinal dystrophy associated with nephronophthisis. Vision Res. 2012;75:88-97.

17. Deacon BS, Lowery RS, Phillips PH, Schaefer GB. Congenital ocular motor apraxia, the NPHP1 gene, and surveillance for nephronophthisis. J AAPOS. 2013;17:332-3.

18. Valente EM, Dallapiccola B, Bertini E. Joubert syndrome and related disorders. Handb Clin Neurol. 2013;113:1879-88.

19. Bredrup C, Saunier S, Oud MM, Fiskerstrand T, Hoischen A, Brackman D, Leh SM, Midtbø M, Filhol E, Bole-Feysot C, Nitschké P, Gilissen C, Haugen OH, Sanders JS, Stolte-Dijkstra I, Mans DA, Steenbergen EJ, Hamel BC, Matignon M, Pfundt R, Jeanpierre C, Boman H, Rødahl E, Veltman JA, Knappskog PM, Knoers NV, Roepman R, Arts HH. Ciliopathies with skeletal anomalies and renal insufficiency due to mutations in the IFT-A gene WDR19. Am J Hum Gene. 2011;89:634-43.

20. Hildebrandt F, Otto E, Rensing C, Nothwang HG, Vollmer M, Adolphs J, Hanusch $\mathrm{H}$, Brandis $\mathrm{M}$. A novel gene encoding an SH3 domain protein is mutated in nephronophthisis type 1. Nat Genet. 1997;17:149-53.

21. Meyer KJ, Davis LK, Schindler EI, Beck JS, Rudd DS, Grundstad AJ, Scheetz TE, Braun TA, Fingert JH, Alward WL, Kwon YH, Folk JC, Russell SR, Wassink TH, Stone EM, Sheffield VC. Genome-wide analysis of copy number variants in AMD. Hum Genet. 2011;129:91-100.

22. Cheung CM, Wong TY. Is age-related macular degeneration a manifestation of systemic disease? New prospects for early intervention and treatment. J Intern Med. 2014;276:140-53. 
23. Sugiyama N, Kohno M, Yokoyama T. Inhibition of the p38 MAPK pathway ameliorates renal fibrosis in an NPHP2 mouse model. Nephrol Dial Transplant. 2012;27:1351-8.

24. Gattone VH 2nd, Sinders RM, Hornberger TA, Robling AG. Late progression of renal pathology and cyst enlargement is reduced by rapamycin in a mouse model of nephronophthisis. Kidney Int. 2009;76:178-82.

25. Chen NX, Moe SM, Eggleston-Gulyas T, Chen X, Hoffmeyer WD, Bacallao RL, Herbert BS, Gattone VH 2nd. Calcimimetics inhibit renal pathology in rodent nephronophthisis. Kidney Int. 2011;80:612-9.
26. Wang S, Dong Z. Primary cilia and kidney injury: current research status and future perspectives. Am J Physiol Renal Physiol. 2013;305:F1085-98.

27. Hamiwka LA, Midgley JP, Wade AW, Martz KL, Grisaru S. Outcomes of kidney transplantation in children with nephronophthisis: an analysis of the North American Pediatric Renal Trials and Collaborative Studies (NAPRTCS) Registry. Pediatr Transplant. 2008;12:878-82. 